\title{
S3-Leitlinie Palliativmedizin
}

\section{Vorsichtig ausgelotet, konsensorientiert}

Die S3-Leitlinie „Palliativmedizin für Patienten mit einer nicht heilbaren Krebserkrankung“ ist verabschiedet und veröffentlicht [www. leitlinienprogramm-onkologie.de]. Gut ist das, weil es bedeutet, dass in der täglichen Praxis, in der klinischen Forschung und bei den Fachgesellschaften so viel Wissen, Erfahrung und Konsens gesammelt wurde, dass erstmals in einem umfangreichen und verbindlichen Papier zusammengefasst werden konnte, was Palliativmedizin heute ist und wie sie betrieben werden soll. Anders formuliert: Die Pionierphase der Palliativmedizin ist (auch) in Deutschland beendet.

Die Leitlinie behandelt in acht Kapiteln die Themen: Grundsätze der Palliativmedizin, Atemnot, Tumorschmerz, Obstipation, Depression, Kommunikation, Sterbephase und Versorgungsstrukturen; weitere sollen folgen: maligne intestinale Obstruktion, Übelkeit und Erbrechen, Schlafstörungen, Wundpflege, Fatigue, Angst, Therapiezielfindung, Umgang mit Todeswunsch.

\section{Sorgfalt und Vorsicht sind sowohl Stärke als auch Schwäche der Leitlinie}

In der Leitlinie wird sehr penibel Satz für Satz festgehalten, auf welcher wissenschaftlichen Evidenz die Empfehlungen gründen und mit welchem Grad der Verbindlichkeit sie gegeben werden. Das gibt Behandlern, Betreuern und Patienten aber auch Kostenträgern mehr Orientierung in einem medizinischen Versorgungsfeld, das zwar eigentlich uralt, aber wissenschaftlich und strukturell noch ganz jung ist. Diese Sorgfalt und Vorsicht ist sowohl die Stärke als auch die Schwäche der Leitlinie. Alles was hier zu lesen ist, ist vorsichtig ausgelotet und auf Konsens orientiert.

Pointierte Aussagen zu kontroversen Themen findet man daher ebenso wenig wie Praxistipps oder ungewöhnliche Maßnahmen in Extremsituationen. Im Kapitel „Atemnot“ beispielsweise wird diese expressis verbis als „unangenehmes Gefühl“ abgehandelt und geradezu verharmlost. Nur beiläufig findet sich in den Erläuterungen ein Hinweis auf Panik und Angst, aber ohne Handlungsempfehlung. Dass Krebspatienten im Extremfall buchstäblich ersticken können, wird nicht erwähnt. An dieser Stelle wäre in einer Leitlinie auch eine Aussage zur Indikation einer palliativen Sedierung fällig. Sie fehlt. Auch im Kapitel Tumorschmerz gibt es solche Lücken: Metamizol/Novaminsulfon ist in Deutschland für sehr viele onkologische Palliativmediziner sicher das wichtigste Analgetikum aus der Gruppe der Nichtopioide. In einigen europäischen Ländern ist es gar nicht zugelassen. Was sagen die Leitlinien dazu? „Metamizol kann als Monotherapie bei leichten Schmerzen und als Kombinationstherapie mit Opioiden bei mittleren und starken Tumorschmerzen als Alternative zu NSAR und Paracetamol eingesetzt werden.“ Mehr nicht. Das ist eher diplomatisch als hilfreich.

Die beschriebene Zurückhaltung aus Genauigkeit und Vorsicht muss allerdings nicht nur ein Nachteil sein. Die Palliativ-

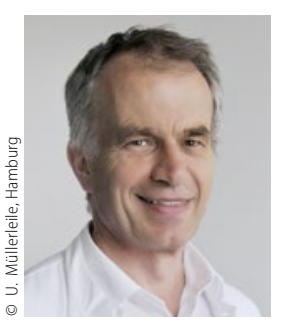

Dr. med. Ulrich Müllerleile

Schwerpunktpraxis Onkologie Hämatologie, Hamburg

umuellerleile@web.de

mediziner müssen sich - bei aller Sympathie, die der Entwicklung ihres Faches in den letzten Jahren öffentlich entgegengebracht wurde - im engeren Bereich des Medizinbetriebes den Respekt der Fachkollegen noch Tag für Tag erkämpfen. Da kann das Streben nach evidenzbasiertem Handeln nur nützen.

Zum Nachteil gerät diese Sorgfalt allerdings dann, wenn beim Lesen der Eindruck entsteht, dass viel Selbstverständliches umständlich und ausführlich ausgebreitet, aber schwierige und praxisrelevante Themen oder Kontroversen umgangen oder auf später verschoben werden:

- Die Notwendigkeit, Palliativmedizin viel früher in die onkologische Versorgung zu integrieren, wird eher implizit und verklausuliert als deutlich angesprochen.

- Brisante Themen, wie die sehr häufig falsch indizierte oder falsch durchgeführte palliative Sedierung, werden mit wenigen unverbindlichen Sätzen abgehandelt.

- Das gesellschaftlich gerade so heiß diskutierte Thema der ärztlichen Sterbebegleitung wurde verschoben. Damit wurde auch eine Chance für eine gesteigerte öffentliche Wahrnehmung der Leitlinie vergeben.

\section{Leitlinien müssen gelesen werden}

Leitlinien müssen aber auch gelesen werden, damit sich der Aufwand lohnt. Es ist zu hoffen, dass es bei der angekündigten Vervollständigung durch eine praxisnahe, präzise, kürzere und problemorientierte Darstellung besser als in dieser ersten Leitlinie gelingt, Kolleginnen und Kollegen zum Lesen anzuregen: Bei einem Expertengespräch der Hamburger Krebsgesellschaft im November 2015 - Teilnehmer waren allesamt aktive Palliativmediziner - stellte sich heraus, dass sechs Monate nach der Veröffentlichung dieser wichtigen Leitlinie nur sehr wenige diese gelesen hatten. Dass das bei der geplanten Fortsetzung nicht so bleibt, liegt auch in der Hand der Autoren.

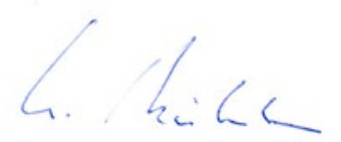

